\title{
Quantum measurement characteristics of a double-dot single electron transistor
}

\author{
HuJun Jiao* and Xin-Qi $\mathrm{Li}^{\dagger}$ \\ State Key Laboratory for Superlattices and Microstructures, Institute of Semiconductors, \\ Chinese Academy of Sciences, P.O. Box 912, Beijing 100083, China \\ JunYan Luo \\ State Key Laboratory for Superlattices and Microstructures, Institute of Semiconductors, \\ Chinese Academy of Sciences, P.O. Box 912, Beijing 100083, China \\ and Department of Chemistry, Hong Kong University of Science and Technology, Kowloon, Hong Kong \\ (Received 30 December 2006; revised manuscript received 2 April 2007; published 26 April 2007)
}

\begin{abstract}
Owing to a few unique advantages, the double-dot single electron transistor has been proposed as an alternative detector for charge states. In this work, we present a further study for its signal-to-noise property, based on a full analysis of the setup configuration symmetry. It is found that the effectiveness of the double-dot detector can approach that of an ideal detector, if the symmetric capacitive coupling is taken into account. The quantum measurement efficiency is also analyzed by comparing the measurement time with the measurementinduced dephasing time.
\end{abstract}

DOI: $10.1103 /$ PhysRevB.75.155333

PACS number(s): 73.63.Kv, 73.23.- b, 03.65.Yz

\section{INTRODUCTION}

Quantum measurement in solid-state mesoscopic systems has attracted considerable interest in the past years. ${ }^{1-7} \mathrm{Be}-$ sides the intensive theoretical work, experimental progresses are, in particular, impressive ${ }^{8-15}$ In these studies, two measurement devices were typically focused on, i.e., the mesoscopic quantum point contact (QPC) and the single electron transistor (SET). Usually, the SET is restricted to the device with a single dot embedded in between the source and drain electrodes. Very recently, the double-dot (DD) SET has been proposed as an alternative charge detector. ${ }^{16-21}$ Compared to the single-dot detector, in addition to the obvious advantage of weakening the requirement of very low temperature, the DD detector may have other advantages such as the following: (i) It can probe the rapid transitions between electrostatically degenerate charge states. ${ }^{17}$ Experimentally, its radiofrequency operation has been demonstrated. ${ }^{18}$ (ii) DD detector is able to probe the entanglement of two qubits. ${ }^{19}$ (iii) Most importantly, DD detector has better immunity against noises. ${ }^{20}$

Owing to the added complexity of the DD detector, better understanding of its measurement dynamics is of interest and seems a timely work at this stage. Very recently, this problem was studied by Gilad and Gurvitz. ${ }^{21}$ The key insight gained in their work is the symmetry property of the setup configuration, which is revealed in terms of the response current of the DD detector in both the time and frequency domains. However, their analysis was based on an extremely asymmetric capacitive coupling configuration, which leads to a conclusion that the DD detector is a sensitive detector, but cannot reach the signal-to-noise ratio of 4 , i.e., the value of an ideal QPC detector.

In this work, we present a further study for the signal-tonoise property of the DD detector, based on a full analysis of the capacitive coupling symmetry. In contrast with Ref. 21, we conclude that the DD detector can approach the signalto-noise ratio of an ideal QPC detector, if the symmetric capacitive coupling setup is taken into account. Moreover, we also analyze the quantum measurement efficiency of the DD detector by comparing the measurement time with the measurement-induced dephasing time. It is found that under the setup configuration that results in the optimal signal-tonoise ratio, the measurement efficiency cannot reach unity (i.e., the value of ideal QPC detector). However, in principle, it can approach unity under proper parametric conditions.

\section{MODEL DESCRIPTION}

As schematically shown in Fig. 1, let us consider a charge qubit measured by a mesoscopic transport device. The charge qubit studied here is modeled by a pair of coupled quantum dots with an extra electron in it, while the detector is the proposed DD single electron transistor. The entire system is described by the following Hamiltonian:

$$
H=H_{0}+H^{\prime}
$$

$$
H_{0}=H_{s}+\sum_{k}\left(\epsilon_{k}^{L} c_{k}^{\dagger} c_{k}+\epsilon_{k}^{R} d_{k}^{\dagger} d_{k}\right)
$$

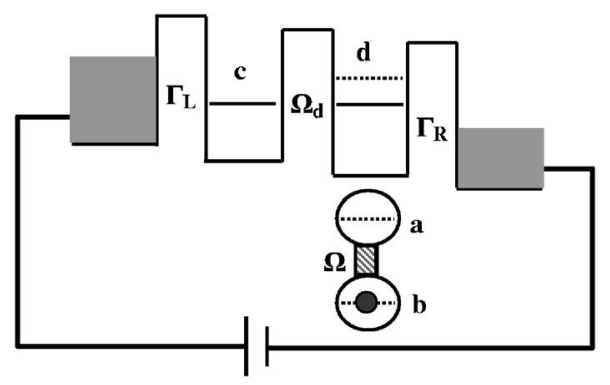

FIG. 1. Schematic setup of using the double-dot single electron transistor to perform quantum measurement of a solid-state qubit. 


$$
\begin{aligned}
H_{s}= & \sum_{i=a, b, c, d} E_{i} a_{i}^{\dagger} a_{i}+\Omega\left(a_{a}^{\dagger} a_{b}+a_{b}^{\dagger} a_{a}\right)+\Omega_{d}\left(a_{c}^{\dagger} a_{d}+a_{d}^{\dagger} a_{c}\right) \\
& +\sum_{i=a, b} \sum_{j=c, d} U_{i j} n_{i} n_{j}+U_{c d} n_{c} n_{d}, \\
H^{\prime}= & \sum_{k}\left(\Omega_{k}^{L} a_{c}^{\dagger} c_{k}+\Omega_{k}^{R} a_{d}^{\dagger} d_{k}+\text { H.c. }\right) \equiv a_{c}^{\dagger} f_{c}+a_{d}^{\dagger} f_{d}+\text { H.c. }
\end{aligned}
$$

In these decomposed Hamiltonians, $a_{a}^{\dagger}\left(a_{a}\right), a_{b}^{\dagger}\left(a_{b}\right), a_{c}^{\dagger}\left(a_{c}\right)$, $a_{d}^{\dagger}\left(a_{d}\right), c_{k}^{\dagger}\left(c_{k}\right)$, and $d_{k}^{\dagger}\left(d_{k}\right)$ are the electron creation (annihilation) operators of the qubit, detector's two central dots, and the reservoirs. In the following treatment, the tunneling Hamiltonian $H^{\prime}$ of the DD detector will be taken as perturbation. The free Hamiltonian in the above, $H_{0}$, consists of the detector's reservoirs, its central two dots, the qubit, and the Coulomb interaction between them.

In this work, we assume that the DD detector works in the strong Coulomb-blockade regime, i.e., there will be at most one more electron occupied in the two dots. Therefore, only the three DD states $|00\rangle,|10\rangle$, and $|01\rangle$ are involved in the transport process. Here, 0 and 1 stand for the vacant and occupied dot states, while their ordering in " $|.$.$\rangle " is from the$ left to the right dot states of the detector. For the qubit, it has two logic states, i.e., the dot states $|a\rangle$ and $|b\rangle$. For the sake of simplicity, we assume that each dot has only one bound state. Intuitively, the measurement principle of the device under study is as follows: if the qubit is in state $|b\rangle$, the two states $|10\rangle$ and $|01\rangle$ of the DD detector are nearly energetically degenerate; while the qubit is in state $|a\rangle$, they will be in off resonance, due to the relatively stronger Coulomb interaction $U_{a d}$. Accordingly, the resultant different output currents of the DD detector can distinguish the qubit states.

\section{$n$-RESOLVED MASTER EQUATION}

In the reduced description, the central dots of the detector and the qubit are the system of interest, and the two reservoirs of the detector are the environment. The first step is to derive a master equation for the system of interest. Moreover, in order to relate the master equation also to the output of the detector, one should obtain an $n$-resolved master equation. Here, $n$ denotes the number of electrons in certain specified time interval that have tunneled through the left or right junction of the transport device. Following the previous work about the master equation, ${ }^{2,5,6,21-23}$ we obtain

$$
\begin{aligned}
\dot{\rho}^{\left(n_{R}\right)}= & -i \mathcal{L} \rho^{\left(n_{R}\right)}-\frac{1}{2}\left\{\left[a_{c}^{\dagger}, A_{c}^{(-)} \rho^{\left(n_{R}\right)}-\rho^{\left(n_{R}\right)} A_{c}^{(+)}\right]+a_{d}^{\dagger} A_{d}^{(-)} \rho^{\left(n_{R}\right)}\right. \\
& \left.+\rho^{\left(n_{R}\right)} A_{d}^{(+)} a_{d}^{\dagger}-\left[a_{d}^{\dagger} \rho^{\left(n_{R}+1\right)} A_{d}^{(+)}+A_{d}^{(-)} \rho^{\left(n_{R}-1\right)} a_{d}^{\dagger}\right]+\text { H.c. }\right\} .
\end{aligned}
$$

Note that throughout this paper, we shall use the unit system of $\hbar=e=k_{B}=1$. Shown above is, in fact, the $n_{R}$-resolved master equation, with $n_{R}$ the number of electrons that have tunneled through the right junction. Similar equation can be carried out for the left-junction specified tunneled electrons. The superoperators in Eq. (2) read $A_{\alpha}^{( \pm)}=C_{\alpha}^{( \pm)}( \pm \mathcal{L}) a_{\alpha}$.
$C_{\alpha}^{( \pm)}( \pm \mathcal{L})$ are the spectral functions of the two reservoirs, which are the Fourier transform of the correlation functions, i.e., $C_{\alpha}^{( \pm)}( \pm \mathcal{L})=\int_{-\infty}^{+\infty} d t C_{\alpha}^{( \pm)}(t) e^{ \pm i \mathcal{L} t}$, with $C_{\alpha}^{(+)}(t)=\left\langle f_{\alpha}^{\dagger}(t) f_{\alpha}\right\rangle$ and $C_{\alpha}^{(-)}(t)=\left\langle f_{\alpha}(t) f_{\alpha}^{\dagger}\right\rangle$.

Note that the Liouvillian $\mathcal{L}$ is defined by $\mathcal{L}(\cdots)$ $=\left[H_{S}, \ldots\right]$. To explicitly carry out the action of its arbitrary function on an operator (e.g., $a_{c}$ or $a_{d}$ ), a convenient way is doing it in the eigenstate basis of $H_{S}$. In this basis, the matrix element of the arbitrary function of $\mathcal{L}$ is obtained by simply replacing $\mathcal{L}$ with the energy difference of the two basis states.

\section{READOUT CHARACTERISTICS}

Note that $\rho^{(n)}$ contains rich information about the measurement. From it, one can obtain the distribution function of the tunneled electron numbers, the output current, and the noise spectrum. Quite clearly, the distribution function reads $P\left(n_{R}, t\right)=\operatorname{Tr}\left[\rho^{n_{R}}(t)\right]$, where the trace is over the states of the system of interest. Then, the current through the right junction is

$$
I_{R}(t)=\sum_{n_{R}} \operatorname{Tr}\left\{n_{R} \dot{\rho}^{\left(n_{R}\right)}\right\}=\frac{1}{2} \operatorname{Tr}\left\{\left[a_{d}^{\dagger} A_{d}^{(-)}-A_{d}^{(+)} a_{d}^{\dagger}\right] \rho(t)+\text { H.c. }\right\},
$$

where $\rho(t)=\Sigma_{n_{R}} \rho^{\left(n_{R}\right)}(t) . \rho(t)$ satisfies the usual unconditional master equation, which can be straightforwardly obtained in this context by summing up Eq. (2) over $n_{R}$. Similar result as Eq. (3) can be obtained for $I_{L}(t)$, the current through the left junction.

Now, we formulate the calculation of the output power spectrum. It is well known that the noise spectrum is a measure of the temporal correlation of the current. The temporal fluctuating currents through the left and right junctions, even in steady state, are not equal to each other. The circuit current, which is typically the measured quantity in most experiments, is a superposition of the left and right currents, i.e., $I(t)=a I_{L}(t)+b I_{R}(t)$. Here, the coefficients $a$ and $b$ satisfy $a$ $+b=1$ and depend on the junction capacitances of the detector. ${ }^{24}$ Note that this capacitive geometry is not necessarily in accordance with the tunnel couplings. For very asymmetric tunnel couplings, the capacitive geometry can be quite symmetric. In what follows, we shall see that this is, in fact, the setup we want to suggest.

In view of the charge conservation, i.e., $I_{L}=I_{R}+\dot{Q}$, where $Q$ is the charge on the central dots, we obtain $I(t) I(0)$ $=a I_{L}(t) I_{L}(0)+b I_{R}(t) I_{R}(0)-a b \dot{Q}(t) \dot{Q}(0)$. Accordingly, the noise spectrum is a sum of three parts,

$$
S(\omega)=a S_{L}(\omega)+b S_{R}(\omega)-a b \omega^{2} S_{Q}(\omega),
$$

where $S_{L / R}(\omega)$ is the noise spectrum of the current through the left (right) junction, and $S_{Q}(\omega)$ characterizes the charge fluctuations on the central dots. For $S_{L / R}(\omega)$, it follows the MacDonald's formula 


$$
S_{\alpha}(\omega)=2 \omega \int_{0}^{\infty} d t \sin \omega t \frac{d}{d t}\left\langle n_{\alpha}^{2}(t)\right\rangle,
$$

where $\left\langle n_{\alpha}^{2}(t)\right\rangle=\Sigma_{n_{\alpha}} n_{\alpha}^{2} \operatorname{Tr} \rho^{\left(n_{\alpha}\right)}(t)=\Sigma_{n_{\alpha}} n_{\alpha}^{2} P\left(n_{\alpha}, t\right)$. With the help of Eq. (2), we further obtain

$$
\frac{d}{d t}\left\langle n_{\alpha}^{2}(t)\right\rangle=\operatorname{Tr}\left[2 \mathcal{J}_{\alpha}^{(-)} N^{\alpha}(t)+\mathcal{J}_{\alpha}^{(+)} \rho+\text { H.c. }\right],
$$

where the particle-number matrix reads $N_{\alpha}(t)$ $\equiv \Sigma_{n_{\alpha}} n_{\alpha} \rho^{\left(n_{\alpha}\right)}(t)$, and the superoperator means

$$
\mathcal{J}_{\alpha}^{( \pm)}(\cdots)=\frac{1}{2}\left[A_{\mu}^{(-)}(\cdots) a_{\mu}^{+} \pm a_{\mu}^{+}(\cdots) A_{\mu}^{(+)}\right] .
$$

In this last equation, $\mu=c$ if $\alpha=L$, and $\mu=d$ if $\alpha=R$.

Following Ref. 25, it will be very convenient to work in the frequency domain. Inserting Eq. (6) into Eq. (5), we obtain

$$
\begin{aligned}
S_{\alpha}(\omega)= & 2 \omega \operatorname{Im}\left[\operatorname { T r } \left(2\left\{\mathcal{J}_{\alpha}^{(-)} \tilde{N}^{\alpha}(\omega)+\left[\mathcal{J}_{\alpha}^{(-)} \tilde{N}^{\alpha}(-\omega)\right]^{\dagger}\right\}\right.\right. \\
& \left.\left.+\left\{\mathcal{J}_{\alpha}^{(+)} \tilde{\rho}(\omega)+\left[\mathcal{J}_{\alpha}^{(+)} \tilde{\rho}(-\omega)\right]^{\dagger}\right\}\right)\right],
\end{aligned}
$$

where $\widetilde{N}^{\alpha}(\omega)=\int_{0}^{\infty} d t N^{\alpha}(t) e^{i \omega t}$, and $\widetilde{\rho}(\omega)=\int_{0}^{\infty} d t \rho^{s t} e^{i \omega t}$. Note that $\rho^{s t}$ is the stationary state density matrix, which is time independent. We thus have $\widetilde{\rho}(\omega)=i \rho^{s t} / \omega$. For $N^{\alpha}(\omega)$, it can be easily obtained by solving a set of algebraic equations after Fourier transforming the equation of motion of $N^{\alpha}(t)$, as have been clearly described in Ref. 25 .

Concerning the charge fluctuations on the central dots, we define the noise spectrum as

$S_{Q}(\omega)=\int_{-\infty}^{\infty} d \tau\langle N(\tau) N+N N(\tau)\rangle e^{i \omega \tau}=4 \operatorname{Re}\left[\int_{0}^{\infty} d \tau S(\tau) e^{i \omega \tau}\right]$,

where we have introduced $S(\tau)=\langle N(\tau) N\rangle$. More explicitly, it can be expressed as $S(\tau)=\operatorname{Tr} \operatorname{Tr}_{B}\left[U^{\dagger}(\tau) N U(\tau) N \rho^{s t} \rho_{B}\right]$, where $U(\tau)=e^{-i H \tau}$, and $N$ is the electron number operator of the central dots of the detector. Using the cyclic property under trace, we obtain $S(\tau)=\operatorname{Tr}[N \sigma(\tau)]$ and $\sigma(\tau)$ $\equiv \operatorname{Tr}_{B}\left[U(\tau) N \rho^{s t} \rho_{B} U^{\dagger}(\tau)\right]$. Obviously, $\sigma(\tau)$ satisfies the same equation of the reduced density matrix $\rho(\tau)$. The only difference is the initial condition, for $\sigma(\tau)$ which is $\sigma(0)=N \rho^{s t}$. Similar to the above, from the equation of motion of $\sigma(\tau)$, its Fourier counterpart $\widetilde{\sigma}(\omega)$ can be straightforwardly carried out. Then, the charge fluctuation spectrum is obtained as $S_{Q}(\omega)=4 \operatorname{Re} \operatorname{Tr}[N \widetilde{\sigma}(\omega)]$.

Based on the above formalism, we now investigate the readout characteristics of the DD detector. The first important quantity to characterize the detector is the visibility, which is defined by $p=|\Delta I| / \bar{I}=2\left|I_{a}-I_{b}\right| /\left(I_{a}+I_{b}\right)$. In Fig. 2, we plot the visibility against the qubit-detector interaction strength $U_{a d}$, by taking the temperature and the dot-dot coupling strength $\Omega_{d}$ of the DD detector as other comparative parameters. By comparing the results shown in Figs. 2(a) and 2(b), it is found that for smaller $\Omega_{d}$ the visibility can more easily approach the ideal value of 2 , by increasing the interaction strength $U_{a d}$. In practice, controlling $U_{a d}$ is difficult.
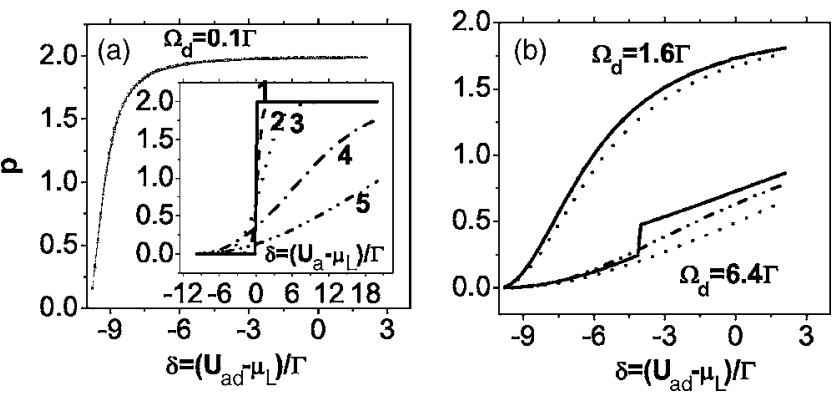

FIG. 2. Tolerance of (not low-enough) finite temperatures of the visibility, $p=|\Delta I| / \bar{I}=2|\Delta I| /\left(I_{a}+I_{b}\right)$, for different interdot couplings of the DD detector: (a) $\Omega_{d} / \Gamma=0.1$ and (b) $\Omega_{d} / \Gamma=1.6$ and 6.4. As a comparison, the result of single-dot SET is plotted in the inset of (a), where $U_{a}$ is the Coulomb interaction between the qubit electron in dot $a$ and the transport electron in the central dot of SET. Results are illustrated for three temperatures for the DD detector, $T / \Gamma=0$, 1.6, and 12.8 (corresponding to the solid, dot-dot-dashed, and dotted lines), while for five temperatures for the single-dot SET, $T / \Gamma$ $=0,0.4,1.6,6.4$, and 12.8 (labeled by "1," "2,"..., "5"). For the DD detector, the results are indistinguishable for small $\Omega_{d}$ as shown in (a); the immunity against temperature will be weakened only for large $\Omega_{d}$, as shown in (b). In contrast, the visibility of the single-dot SET is affected by temperatures much more sensitively. In the whole calculations throughout the work, we assume that $\Gamma_{L}=\Gamma$, and use $\Gamma$ as the energy unit. For the result shown in this figure, we chose $\Gamma_{R}=\Gamma$. Other parameters are adopted as $E_{c}=E_{d}=0, U_{a c}$ $=U_{b c}=U_{b d}=0, \mu_{L}=10 \Gamma$, and $\mu_{R}=-10 \Gamma$.

However, engineering $\Omega_{d}$ is relatively easy, which opens a way to enhance the visibility as revealed in Fig. 2. In this context, one should also notice another major advantage of the DD detector, say, its better tolerance to finite temperatures. From Fig. 2, we see that the finite temperature does not sensitively affect the operation of the DD detector under proper parametric conditions, particularly for small $\Omega_{d}$ as shown in Fig. 2(a). Contrary to that, in the inset of Fig. 2(a), the result of single-dot detector is presented, of which the visibility sensitively depends on the temperature. All these features can be easily understood in terms of resonant tunneling through the double dots and single dot, respectively.

In addition to the visibility, the quality of a quantum detector is well characterized by the signal-to-noise ratio, i.e., the peak-to-pedestal ratio of the output power spectrum. Not as in Ref. 21, where the capacitively asymmetric coupling model, i.e., with $a=0$ and $b=1$, was taken into account, below we calculate the noise spectrum in general under arbitrary capacitive couplings. In particular, the symmetric coupling, say, $a=b=1 / 2$, will be focused. Notably, from Fig. 3(a) we find that the peak-to-pedestal ratio is sensitively affected by the tunnel rate $\Gamma_{R}$ of the right junction, where the measured qubit is placed nearby. This feature is in qualitative agreement with that found by Gilad and Gurvitz, ${ }^{21}$ although a different definition of the signal-to-noise ratio was employed there.

In Fig. 3(b), we show the effect of the capacitive coupling symmetry. It is found that the signal-to-noise ratio will reach the maximum at the symmetric coupling, i.e., when $a=1 / 2$. This is because the charge-number fluctuation on the two dots of the detector has negative contribution to the noise 

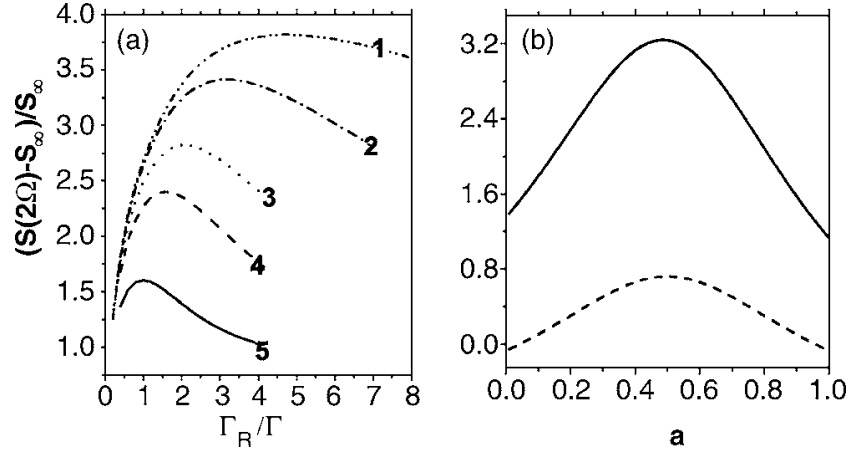

FIG. 3. Configuration symmetry dependence of the peak-topedestal ratio of the output power spectrum: (a) $\Gamma_{R}$ dependence and (b) capacitive coupling dependence. Note that in (b) the parameter $a$ characterizes the capacitive coupling symmetry (see the main text for its more detailed explanation). The major parameters are the same as in Fig. 2, except for the differences as follows: In present result, it is assumed that $\Omega=\Omega_{d}=0.2 \Gamma$, and the temperature $T=0$. In (a), we assume a symmetric configuration of capacitive coupling, i.e., $a=1 / 2$, and assume the Coulomb interaction strengths as (1) $U_{a d} / \Gamma=12$, (2) $U_{a d} / \Gamma=6$, (3) $U_{a d} / \Gamma=3$, (4) $U_{a d} / \Gamma=2$, and (5) $U_{a d} / \Gamma=1$. In (b), in addition to the result depicted by the solid curve, which corresponds to the suggested location of the qubit nearby the right dot of the DD detector (as schematically shown in Fig. 1), we also plot the result by the dashed curve for the result of configuration with the qubit nearby the left dot. For the former configuration, $U_{a c}=0$ and $U_{a d}=6 \Gamma$, while for the latter, $U_{a d}=0$ and $U_{a c}=6 \Gamma$. For both configurations, $\Gamma_{R}=2 \Gamma$ is commonly used.

spectrum, thus largely suppresses the background noise. As a consequence, the peak-to-pedestal ratio is enhanced for more symmetric coupling and reaches the maximum at $a=1 / 2$. In Fig. 3(b), the solid (dashed) curve corresponds to the result of the measured qubit next to the right (left) dot of the DD detector. This remarkable difference reflects another interesting symmetry effect of the setup configuration.

Note that $\Gamma_{R}$ and $\Omega_{d}$ are two controllable parameters in practice. We thus replot the signal-to-noise ratio versus the scaled $\Gamma_{R}$ and $U_{a d}$ by $\Omega_{d}$ in order to gain the entire landscape more clearly, as shown in Fig. 4. In this context, we remark that the peak-to-pedestal ratio of the DD detector can approach the upper limit of 4 of the ideal QPC detector, ${ }^{26}$ under proper parametric conditions as indicated by Fig. 4. This conclusion is in contrast with that by Gurvitz and co-workers. ${ }^{6,21}$ There, it was concluded that both the singledot and double-dot detectors are only sensitive measurement devices (i.e., with desirable visibility), but cannot reach the effectiveness of an ideal QPC detector. By tilting the tunnel coupling such that $\Gamma_{R} \gg \Gamma_{L}$, Gurvitz and co-workers found that the signal-to-noise ratio can be considerably enhanced. However, their calculation was restricted to the capacitively asymmetric coupling model, i.e., with $a=0$ and $b=1$. In this case, the upper limit of the signal-to-pedestal ratio is 2 . Here, as clearly shown by Fig. 3(b), our calculation shows that under the symmetric condition $a=b=1 / 2$ the signal-topedestal ratio is maximal and can, in principle, approach the value of 4, which is the upper limit of the ideal QPC detector. $^{26}$

As a brief summary, in the above we revealed three types of configuration dependence: (i) left-versus-right location of

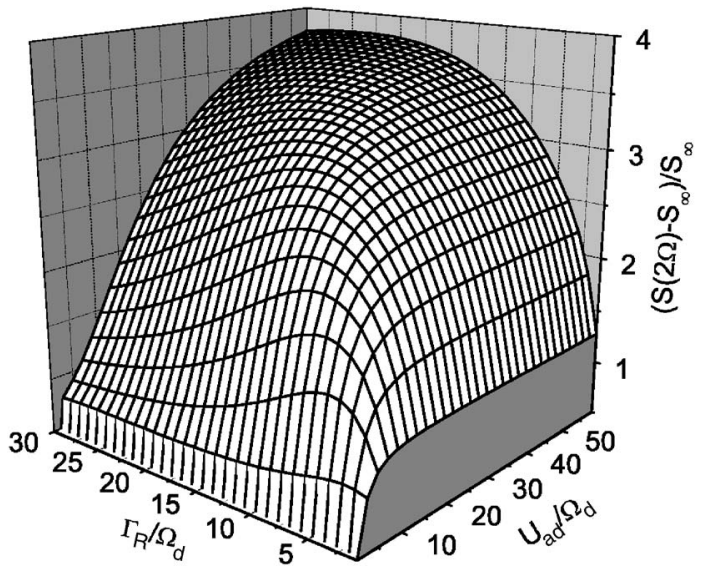

FIG. 4. Three-dimensional plot of the peak-to-pedestal ratio of the output power spectrum as a function of $\Gamma_{R}$ and $U_{a d}$. Relevant parameters are the same as in Fig. 3(a).

qubit with respect to the quantum dots of the DD detector, (ii) relative coupling to the right electrode (i.e., $\Gamma_{R}$ dependence), and (iii) capacitive coupling. While (iii) was resolved in terms of the role of the central charge-number fluctuations in Eq. (4), we would like to elaborate on (i) and (ii) further as follows.

If the qubit is next to the right dot of the DD detector, the interacting time is relatively shorter than the one when the qubit locates nearby the left dot. As a result, smaller backaction induced dephasing rate is anticipated from general consideration, which in turn results in the larger signal-tonoise ratio. Similar reasoning can partially apply to the $\Gamma_{R}$ dependence in Fig. 3(a). However, in addition to the interacting time, the current through the detector, which influences the interacting strength, would also affect the backaction dephasing. For the DD detector, the current difference associated with different qubit states, which is nothing but the signal, shows a turnover behavior with maximum at $\Gamma_{R}$ $=2 \sqrt{2} \Omega_{d}$. Explicit expression is referred to Eq. (4.4) in Ref. 27; see also Eq. (11) in the next section of the present work. The dephasing rate shown in Fig. 5(a) largely follows the behavior of the signal current. With both the dephasing rate and the signal current in mind, the $\Gamma_{R}$ dependence in Fig. 3(a) can be accordingly understood. Note that the small back-action dephasing tends to enhance the signal-to-noise ratio, while in contrast the small signal current would reduce it. The particular line shape of the signal-to-noise ratio versus the $\Gamma_{R}$ is thus a result of these two competing effects, which lead to its turnover behavior and the optimal $\Gamma_{R}$ differing from the dephasing rate in Fig. 5(a).

\section{DEPHASING AND MEASUREMENT TIMES}

In the orthodox Copenhagen postulate for quantum measurement, the measured wave function collapses onto one of the eigenstates of the observable instantaneously. In contrast to that, the wave-function collapse in real device must need some time, i.e., the measurement time. On the other hand, during the collapsing process, dephasing between the superposed wave-function components must take place before 


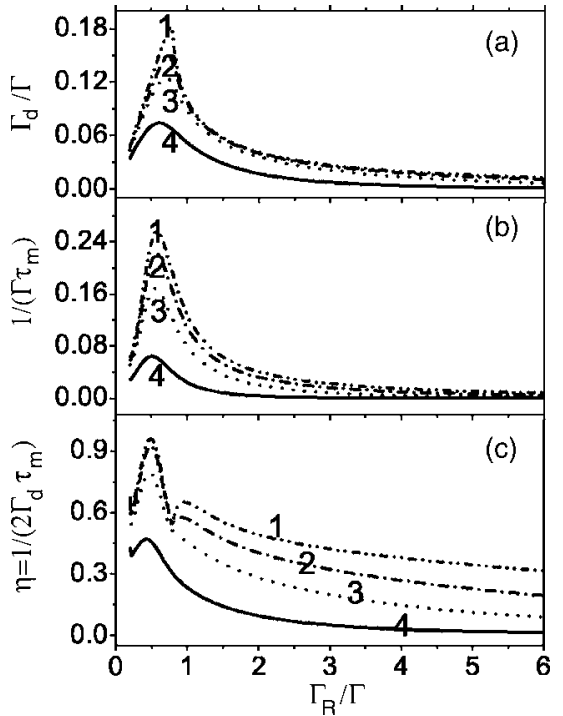

FIG. 5. $\Gamma_{R}$ dependence (i.e., asymmetric effect) of (a) the dephasing rate, (b) the measurement time, and (c) the quantum measurement efficiency. Coulomb interaction strengths: (1) $U_{a d} / \Gamma=12$, (2) $U_{a d} / \Gamma=6$, (3) $U_{a d} / \Gamma=3$, and (4) $U_{a d} / \Gamma=1$. Other relevant parameters are the same as in Fig. 3(a).

reading out the result. Therefore, the ratio of the dephasing time to the measurement time is another deep criterion to characterize the efficiency of quantum measurement. In the following, we carry out a quantitative analysis for the DD detector.

The analysis is also based on the $n$-resolved master equation. Since in this context we are interested in the measurement-induced collapse of wave function, we consider the measurement of the idle state of the qubit. We thus set $\Omega=0$, i.e., switch off the qubit state oscillation. Accordingly, all the mixing terms, i.e., those proportional to $\Omega$, disappear in Eq. (2). The density matrix of the system factorizes into three independent groups. Furthermore, we restrict our analysis to zero temperature, and assume that $E_{i}$ $=0$ for $i=a, b, c$, and $d$ and $U_{a c}=U_{b c}=U_{b d}=0$. By Fourier transforming the resultant $n$-resolved master equation, i.e., defining $\rho(k, t)=\sum_{n_{R}} \rho^{\left(n_{R}\right)}(t) e^{i n_{R} k}$, we obtain

$$
\begin{gathered}
\dot{\rho}_{a a}^{00}=-\Gamma_{L} \rho_{a a}^{00}+\Gamma_{R} e^{i k} \rho_{a a}^{22}, \\
\dot{\rho}_{a a}^{11}=-i \Omega_{d}\left[\rho_{a a}^{21}-\rho_{a a}^{12}\right]+\Gamma_{L} \rho_{a a}^{00}, \\
\dot{\rho}_{a a}^{22}=-i \Omega_{d}\left[\rho_{a a}^{12}-\rho_{a a}^{21}\right]-\Gamma_{R} \rho_{a a}^{22}, \\
\dot{\rho}_{a a}^{12}=i U_{a d} \rho_{a a}^{12}-i \Omega_{d}\left[\rho_{a a}^{22}-\rho_{a a}^{11}\right]-\frac{1}{2} \Gamma_{R} \rho_{a a}^{12}, \\
\dot{\rho}_{b b}^{00}=-\Gamma_{L} \rho_{b b}^{00}+\Gamma_{R} e^{i k} \rho_{b b}^{22}, \\
\dot{\rho}_{b b}^{11}=-i \Omega_{d}\left[\rho_{b b}^{21}-\rho_{b b}^{12}\right]+\Gamma_{L} \rho_{b b}^{00}, \\
\dot{\rho}_{b b}^{22}=-i \Omega_{d}\left[\rho_{b b}^{12}-\rho_{b b}^{21}\right]-\Gamma_{R} \rho_{b b}^{22},
\end{gathered}
$$

$$
\begin{gathered}
\dot{\rho}_{b b}^{12}=-i \Omega_{d}\left[\rho_{b b}^{22}-\rho_{b b}^{11}\right]-\frac{1}{2} \Gamma_{R} \rho_{b b}^{12}, \\
\dot{\rho}_{a b}^{00}=-\Gamma_{L} \rho_{a b}^{00}+\Gamma_{R} e^{i k} \rho_{a b}^{22}, \\
\dot{\rho}_{a b}^{11}=-i \Omega_{d}\left[\rho_{a b}^{21}-\rho_{a b}^{12}\right]+\Gamma_{L} \rho_{a b}^{00}, \\
\dot{\rho}_{a b}^{22}=-i U_{a d} \rho_{a b}^{22}-i \Omega_{d}\left[\rho_{a b}^{12}-\rho_{a b}^{21}\right]-\Gamma_{R} \rho_{a b}^{22}, \\
\dot{\rho}_{a b}^{12}=-i \Omega_{d}\left[\rho_{a b}^{22}-\rho_{a b}^{11}\right]-\frac{1}{2} \Gamma_{R} \rho_{a b}^{12}, \\
\dot{\rho}_{a b}^{21}=-i U_{a d} \rho_{a b}^{21}-i \Omega_{d}\left[\rho_{a b}^{11}-\rho_{a b}^{22}\right]-\frac{1}{2} \Gamma_{R} \rho_{a b}^{21} .
\end{gathered}
$$

We see that these equations split into three groups, i.e., Eqs. (10a)-(10d), Eqs. (10e)-(10h), and Eqs. (10i)-(10m). Here, the density-matrix elements $\rho_{m n}^{i j}=\langle i m|\rho| j n\rangle .|i\rangle$ and $|j\rangle$ denote the occupation states of the DD detector, i.e., $|0\rangle \equiv|00\rangle,|1\rangle$ $\equiv|10\rangle$, and $|2\rangle \equiv|01\rangle$, respectively, while $|m\rangle$ and $|n\rangle$ denote the qubit states $|a\rangle$ and $|b\rangle$.

We now consider the characteristic solutions of the above three groups of equations, i.e., solutions proportional to $e^{i \omega t}$. Technically, for each group of Eqs. (10), we can obtain five eigenvalues. For small values of $k \ll 1$, from the former two groups of Eqs. (10) we obtain the smallest two eigenvalues $\omega_{j}(k)=\left(k+\frac{1}{2} i f^{j} k^{2}\right) \Gamma^{j}$, with $j=a$ and $b$, respectively, which are most relevant to present analysis. $\Gamma^{j}$ are the wave-packet group velocities, which actually correspond to the stationary currents $I_{j}$, with respect to the qubit in state $|j\rangle ; f^{j}$ are the respective Fano factors. Explicitly, from Eqs. (10a)-(10h), $\Gamma^{j}$ and $f^{j}$ are obtained as

$$
\begin{gathered}
\Gamma^{j}=\frac{\Omega_{d}^{2} \Gamma_{R}}{\left(\frac{\Gamma_{R}^{2}}{4}+U_{j}^{2}\right)+\Omega_{d}^{2} \Gamma_{R}\left(\frac{1}{\Gamma_{L}}+\frac{2}{\Gamma_{R}}\right)} \equiv \frac{\Omega_{d}^{2} \Gamma_{R}}{A_{j}}, \\
f^{j}=1+\frac{2 \Omega_{d}^{2}}{A_{j}}\left[2-\frac{\Gamma_{R}^{2}+\left(1+\frac{\Gamma_{R}}{\Gamma_{L}}\right)\left(\frac{\Gamma_{R}^{2}}{4}+U_{j}^{2}+4 \Omega_{d}^{2}\right)}{A_{j}}\right] .
\end{gathered}
$$

Here, the Coulomb interaction energy $U_{a}=U_{a d}$, and $U_{b}=0$, with the convention that the two dot states of the DD detector are in resonance if the qubit is in state $|b\rangle$ and in off resonance by an energy $U_{a}=U_{a d}$ if the qubit is in state $|a\rangle$. Quantitatively, the measurement time can be defined as the required time for signal-to-noise ratio approaching unity. This condition leads to ${ }^{3,4}$

$$
t_{m}=\left(\frac{\sqrt{2 f^{a} \Gamma^{a}}+\sqrt{2 f^{b} \Gamma^{b}}}{\Gamma^{a}-\Gamma^{b}}\right)^{2} .
$$

The dephasing time can be obtained by analyzing Eqs. (10i)-(10m) for $k=0$. Similarly, solve the (five) eigenvalues $\lambda_{i}$ of these equations, then determine the dephasing time in terms of $t_{d}=\max \left[\operatorname{Im} \lambda_{\mathrm{i}}\right]^{-1}$. More importantly, the quantum 
measurement efficiency is obtained via $\eta=1 /\left(2 \Gamma_{d} t_{m}\right)$, where $\Gamma_{d}=1 / t_{d}$.

In Fig. 5, we plot the $\Gamma_{R}$ dependence of the measurement time, dephasing rate, and the quantum efficiency of measurement. At the end of the previous section, we have explained the $\Gamma_{R}$ dependence of the signal-to-noise ratio in terms of dephasing rate and signal current, and pointed out that the dephasing rate is roughly proportional to the signal current, which is now depicted in Fig. 5(a). From the general viewpoint of quantum measurement, the measurement rate, i.e., the rate of information gain, should follow the back-action dephasing rate. This is shown in Fig. 5(b).

The quantum measurement efficiency, which is the ratio of the dephasing time and the measurement time, is shown in Fig. 5(c). We notice that it does not well match the behavior of the signal-to-noise ratio in Fig. 3(a), although both have maxima at proper (different) $\Gamma_{R}$. This feature is not surprising, since the quantum measurement efficiency is anyhow an alternative criterion to qualify the measurement process. That is, it describes how fast the information is gained against the back-action dephasing. ${ }^{28}$

Remarkably, in contrast with the usual statement that the single electron transistor is not an ideal detector, ${ }^{1,3}$ it is found here that the double-dot SET can approximately reach the quantum limit of efficiency under appropriate parametric conditions [see Fig. 5(c)]. However, these parametric conditions do not simultaneously promise the maximal signal-tonoise ratio. It is noticed that the comprehensive work by Clerk et al. had focused on the measurement efficiency of quantum scattering detectors. ${ }^{28,29}$ Using the scattering matrix formalism, general conditions for quantum limited measurements were carried out. Unfortunately, it is not convenient, if not possible, to apply the scattering matrix formalism to the SET-type detectors. Following the line of Clerk et al. especially using the concept of information gain and loss, further elaboration on the quantum limit of efficiency found here is interesting and an open question for future work.

\section{CONCLUSION AND DISCUSSIONS}

To summarize, we have presented a study for the quantum measurement characteristics of double-dot SET. The study was based on a full analysis of the setup configuration geometries, i.e., in terms of the tunneling strengths, capacitive couplings, and the location of the qubit with respect to the DD detector. We found that the DD detector can approach the signal-to-noise ratio of an ideal QPC detector, provided the symmetric capacitive coupling is taken into account. The measurement time, the back-action dephasing time, and the measurement efficiency were calculated. It was found that the quantum limit of efficiency can be reached under proper parametric conditions, although they differ from the ones for obtaining the maximal signal-to-noise ratio.

Finally, we make a few remarks on issues relevant to the present work. In Ref. 30, the measurement properties of the superconducting SET (SSET) were analyzed, where both the coherent Cooper-pair tunneling and the quasiparticle tunneling were taken into account to contribute the measurement current. It was concluded that the Cooper-pair resonance process allows for a much better measurement than a similar nonsuperconducting SET, and can approach the quantum limit of efficiency under proper parametric conditions. In our opinion, the advantages of the SSET are largely a consequence of the coherent tunneling of Cooper pairs, a unique nature of superconductors.

About the nonsuperconducting SET, such as our semiconductor DD detector, we do not expect that higher-order tunnel processes can considerably influence or improve the measurement efficiency. Higher-order (e.g., cotunneling) contribution, which leads to small detection current, was calculated in the Coulomb-blockade regime of SSET, ${ }^{31}$ and was shown to have minor effect on the measurement effectiveness, say, the signal-to-noise ratio.

It has come into our attention that the nonperturbative treatment for strong qubit-detector coupling and arbitrarily strong transmission detector has been recently an attractive research subject. ${ }^{32,33}$ While in these studies the detector is a QPC, similar analysis for SET-type detectors might be an interesting subject of future work. However, typical experiments such as those performed by Marcus and co-workers did not imply strong couplings of the double quantum dots (QDs) with the transport electrodes. ${ }^{34,35}$ In these experiments, the charge configurations of coupled QDs were probed by techniques such as the nearby QPC or direct transport spectroscopy. In order to make the charge states of the coupled QDs well defined, the couplings of the double QDs with the external (transport) electrodes should be relatively weak.

\section{ACKNOWLEDGMENTS}

We thank S. A. Gurvitz for helpful discussion, which drew our attention to the double-dot detector and initiated the present study. This work was supported by the National Natural Science Foundation of China under Grants No. 60425412 and No. 90503013, the Major State Basic Research Project under Grant No. 2006CB921201, and the Research Grants Council of the Hong Kong Government.

\footnotetext{
*Electronic address: jiaohujun@semi.ac.cn

$\dagger$ Electronic address: xqli@red.semi.ac.cn

${ }^{1}$ M. H. Devoret and R. J. Schoelkopf, Nature (London) 406, 1039 (2000).

${ }^{2}$ A. Shnirman and G. Schön, Phys. Rev. B 57, 15400 (1998).
}

${ }^{3}$ Y. Makhlin, G. Schön, and A. Shnirman, Rev. Mod. Phys. 73,
357 (2001).
${ }^{4}$ I. L. Aleiner, N. S. Wingreen, and Y. Meir, Phys. Rev. Lett. 79,
3740 (1997).
${ }^{5}$ S. A. Gurvitz and Ya. S. Prager, Phys. Rev. B 53, 15932 (1996). 
${ }^{6}$ S. A. Gurvitz and G. P. Berman, Phys. Rev. B 72, 073303 (2005).

${ }^{7}$ Hsi-Sheng Goan, G. J. Milburn, H. M. Wiseman, and He Bi Sun, Phys. Rev. B 63, 125326 (2001).

${ }^{8}$ E. Buks, R. Schuster, M. Heiblum, D. Mahalu, and V. Umansky, Nature (London) 391, 871 (1998).

${ }^{9}$ R. J. Schoelkopf, P. Wahlgren, A. A. Kozhevnikov, P. Delsing, and D. E. Prober, Science 280, 1238 (1998).

${ }^{10}$ M. Macucci, M. Gattobigio, and G. Iannaccone, J. Appl. Phys. 90, 6428 (2001).

${ }^{11}$ W. G. van derWiel, S. De Franceschi, J. M. Elzerman, T. Fujisawa, S. Tarucha, and L. P. Kouwenhoven, Rev. Mod. Phys. 75, 1 (2003).

${ }^{12}$ J. M. Elzerman, R. Hanson, L. H. Willems van Beveren, B. Witkamp, L. M. K. Vandersypen, and L. P. Kouwenhoven, Nature (London) 430, 431 (2004).

${ }^{13}$ M. Xiao, I. Martin, E. Yablonovitch, and H. W. Jiang, Nature (London) 430, 435 (2004).

${ }^{14}$ S. Gustavsson, R. Leturcq, B. Simovic, R. Schleser, P. Studerus, T. Ihn, K. Ensslin, D. C. Driscoll, and A. C. Gossard, Phys. Rev. B 74, 195305 (2006).

${ }^{15}$ T. Fujisawa, T. Hayashi, R. Tomita, and Y. Hirayama, Science 312, 1634 (2006).

${ }^{16}$ M. Gattobigio, G. Iannaccone, and M. Macucci, Phys. Rev. B 65, 115337 (2002).

${ }^{17}$ R. Brenner, A. D. Greentree, and A. R. Hamilton, Appl. Phys. Lett. 83, 4640 (2003).

${ }^{18}$ R. Brenner, T. M. Buehler, and D. J. Reilly, J. Appl. Phys. 97, 034501 (2005).
${ }^{19}$ T. Tanamoto and X. Hu, Phys. Rev. B 69, 115301 (2004).

${ }^{20}$ Tamás Geszti and József Zsolt Bernád, Phys. Rev. B 73, 235343 (2006).

${ }^{21}$ T. Gilad and S. A. Gurvitz, Phys. Rev. Lett. 97, 116806 (2006).

${ }^{22}$ X. Q. Li, P. Cui, and Y. J. Yan, Phys. Rev. Lett. 94, 066803 (2005).

${ }^{23}$ X. Q. Li, J. Y. Luo, Y. G. Yang, P. Cui, and Y. J. Yan, Phys. Rev. B 71, 205304 (2005).

${ }^{24}$ Ya. M. Blanter and M. Büttiker, Phys. Rep. 336, 1 (2000).

${ }^{25}$ J. Y. Luo, X. Q. Li, and Y. J. Yan, arXiv:cond-mat/0603163 (unpublished).

${ }^{26}$ A. N. Korotkov, Phys. Rev. B 63, 085312 (2001).

${ }^{27}$ S. A. Gurvitz, Phys. Rev. B 56, 15215 (1997).

${ }^{28}$ A. A. Clerk, S. M. Girvin, and A. D. Stone, Phys. Rev. B 67, 165324 (2003).

${ }^{29}$ A. A. Clerk and A. D. Stone, Phys. Rev. B 69, 245303 (2004).

${ }^{30}$ A. A. Clerk, S. M. Girvin, A. K. Nguyen, and A. D. Stone, Phys. Rev. Lett. 89, 176804 (2002).

${ }^{31}$ J. Kinnunen, P. Törmä, and J. P. Pekola, Phys. Rev. B 68, 020506(R) (2003).

${ }^{32}$ D. V. Averin and E. V. Sukhorukov, Phys. Rev. Lett. 95, 126803 (2005).

${ }^{33}$ I. Snyman and Y. V. Nazarov, arXiv:cond-mat/0701017 (unpublished).

${ }^{34}$ J. R. Petta, A. C. Johnson, C. M. Marcus, M. P. Hanson, and A. C. Gossard, Phys. Rev. Lett. 93, 186802 (2004).

${ }^{35}$ A. C. Johnson, J. R. Petta, C. M. Marcus, M. P. Hanson, and A. C. Gossard, Phys. Rev. B 72, 165308 (2005). 